\title{
Vertigo, CTCAE
}

National Cancer Institute

\section{Source}

National Cancer Institute. Vertigo, CT CAE. NCI Thesaurus. Code C143935.

A disorder characterized by a sensation as if the external world were revolving around the patient (objective vertigo) or as if he himself were revolving in space (subjective vertigo). 